\title{
Transcriptional Regulation of Osteoblasts
}

\author{
RENNY T. FRANCESCHI, ${ }^{a}$ CHUNXI GE,${ }^{a}$ GUOZHI XIAO,${ }^{b}$ \\ HERNAN ROCA, ${ }^{c}$ AND DI JIANG ${ }^{a}$ \\ ${ }^{a}$ University of Michigan School of Dentistry, Ann Arbor, Michigan, USA \\ ${ }^{b}$ University of Pittsburg School of Medicine, Pittsburg, Pennsylvania, USA \\ ${ }^{c}$ University of Michigan School of Medicine and Cancer Center, Ann Arbor, \\ Michigan, USA
}

\begin{abstract}
The differentiation of osteoblasts from mesenchymal precursors requires a series of cell fate decisions controlled by a hierarchy of transcription factors. Among these are RUNX2, Osterix (OSX), ATF4, and a large number of nuclear coregulators. During bone development, initial RUNX2 expression coincides with the formation of mesenchymal condensations well before the branching of chondrogenic and osteogenic lineages. Given that RUNX2 is expressed so early and participates in several stages of bone formation, it is not surprising that it is subject to a variety of controls. These include regulation by nuclear accessory factors and posttranslational modification, especially phosphorylation. Specific examples of RUNX2 regulation include interactions with DLX proteins and ATF4 and phosphorylation by the ERK/MAP kinase pathway. RUNX2 is regulated via phosphorylation of critical serine residues in the $\mathrm{P} / \mathrm{S} / \mathrm{T}$ domain. MAPK activation of RUNX2 was also found to occur in vivo. Transgenic expression of constitutively active MEK1 in osteoblasts accelerated skeletal development while a dominant-negative MEK1 retarded development in a RUNX2-dependent manner. These studies allow us to begin understanding the complex mechanisms necessary to finetune bone formation in response to extracellular stimuli including ECM interactions, mechanical loads, and hormonal stimulation.
\end{abstract}

KEYWORDS: bone; osteoblast; transcription; RUNX2; extracellular matrix

Osteoblast and chondrocyte differentiation is controlled by a hierarchy of transcription factors that are expressed in a defined temporal sequence (FIG. 1). RUNX2, an essential factor for bone and hypertrophic cartilage formation, is expressed very early in skeletal development, first appearing with the

Address for correspondence: Dr. Renny T. Franceschi, Department of Periodontics and Oral Medicine, University of Michigan School of Dentistry, 1011 N. University Ave. Ann Arbor, MI 481091078. Voice: 734-763-7381; fax 734-763-5503.

rennyf@umich.edu

Ann. N.Y. Acad. Sci. 1116: 196-207 (2007). (C) 2007 New York Academy of Sciences. doi: 10.1196/annals.1402.081 


\section{Transcription Factor Control of Skeletal Lineages}

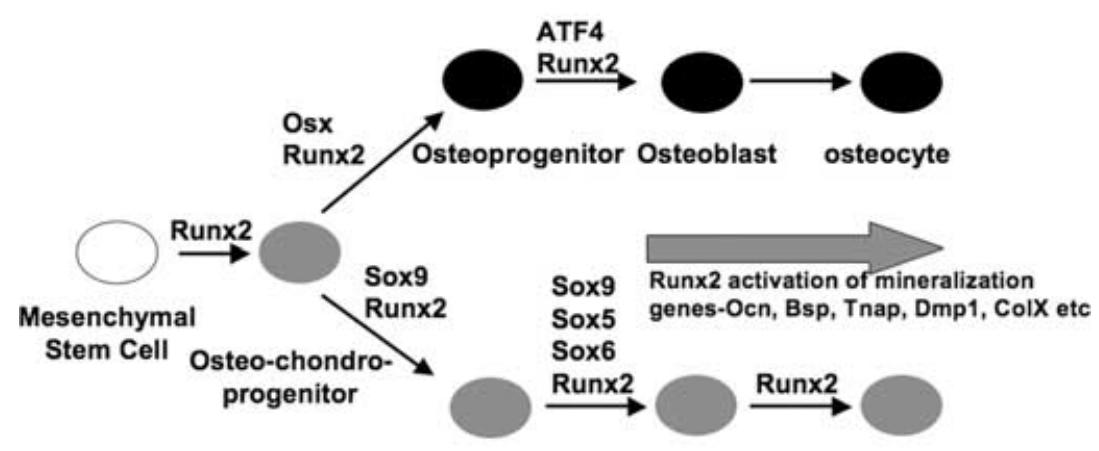

Chondroblast Chondrocyte Hypertrophic

Chondrocyte

FIGURE 1. Transcription factor control of skeletal lineages. Major transcription factors that, based on genetic studies, are involved in osteoblast and chondrocyte differentiation are included in this chart. Also shown is the sequential nature of transcription factor expression with RUNX2 persisting throughout osteoblast and chondrocyte lineages (see text).

formation of mesenchymal condensations in areas destined to become bone and persisting through subsequent stages of bone formation. ${ }^{1}$ Several other transcription factors function together with RUNX2 to move cells down chondrocyte or osteoblast lineages. For osteoblasts, this is accomplished by Osterix (Osx), which commits osteochondroprogenitor cells to the osteoblast lineage. ${ }^{2}$ Subsequently, another factor, ATF4, controls the transcriptional activity of mature osteoblasts. ${ }^{3}$ RUNX2 also participates in the chondrogenic lineage. However, at early stages, it is likely suppressed by the chondrocyte-specific factors, Sox 8/9. ${ }^{4}$ However, Sox factors are downregulated to permit RUNX2 to coordinate chondrocyte hypertrophy. Evidence for this model largely comes from genetic studies showing bone phenotypes of increasing severity as Runx2, Osx, or Atf4 are knocked out. Thus, skeletal development in Runx2-deficient embryos fails to progress beyond the cartilage anlage stage. ${ }^{5,6}$ Fetuses, which die at birth of respiratory failure, have no detectable mineral in either bones or cartilage. In contrast, Osx -/- embryos have hypertrophic cartilage and normal levels of RUNX2, suggesting that this factor is downstream of RUNX2. ${ }^{2}$ ATF4 -/- mice have a milder phenotype consistent with this factor having a regulatory function in bone formation. ${ }^{3}$

An interesting feature of this scheme is that most of the known RUNX2 target genes are only expressed at specific stages of chondrogenic or osteogenic lineages, yet RUNX2 is present throughout both lineages. RUNX2 mRNA is first expressed at E9.5, peaks at E12.5 well before any mineralization has occurred, and continues to be present through the later stages of development. ${ }^{1}$ This essentially constitutive expression of RUNX2 once skeletal development 
(A)

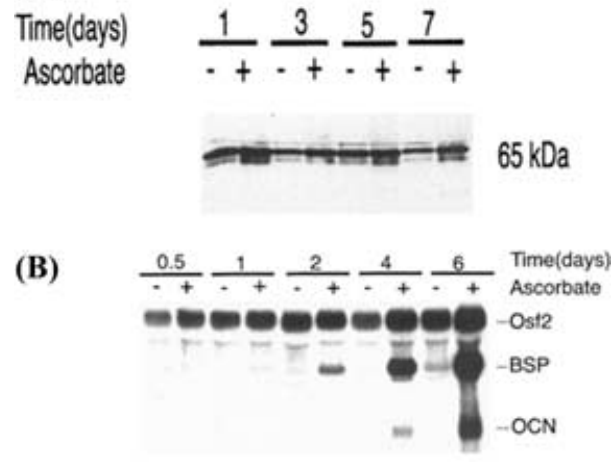

(C)

(D)

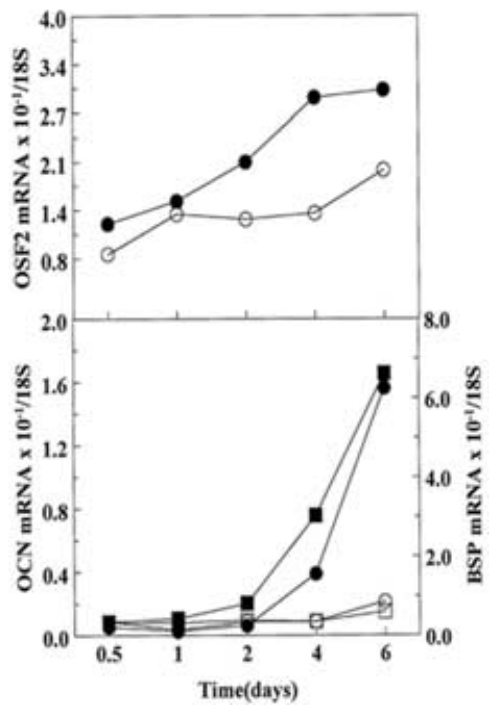

FIGURE 2. RUNX2 protein levels are not well correlated with transcriptional activity. MC3T3-E1 clone 4 preosteoblast cells were grown in control (-) or ascorbate-containing medium (+). At the times indicated, RUNX2 protein levels were determined by Western blotting (A) while Runx2 (Osf2), Ocn and Bsp mRNA levels (B-D) were measured on Northern blots (B) and quantified by densitometry. (C) Runx2; (D) Ocn and Bsp. Legend: open symbols $=$ control; closed symbols $=$ ascorbate. For panel D, Ocn mRNA (m, 1), Bsp mRNA (o, n). From Xiao et al. $1998 .^{7}$

has commenced implies that other factors or signals must be regulating its activity. FIGURE 2 shows an example of how other factors can regulate RUNX2 activity. In this experiment, MC3T3-E1 preosteoblast cells were induced to differentiate by growth in ascorbic acid, thereby allowing cells to secrete a collagenous ECM. Over time, as ECM accumulates, there is a dramatic induction of osteoblast marker genes, such as bone sialoprotein and osteocalcin, 


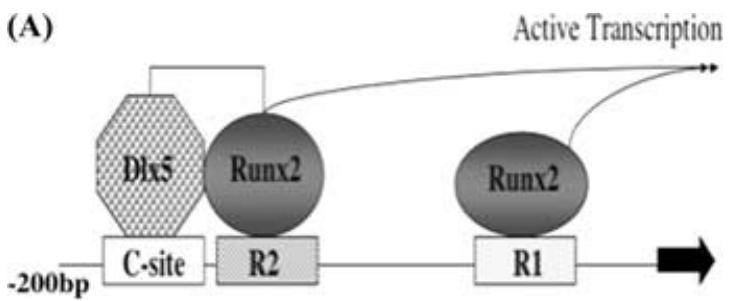

(B)

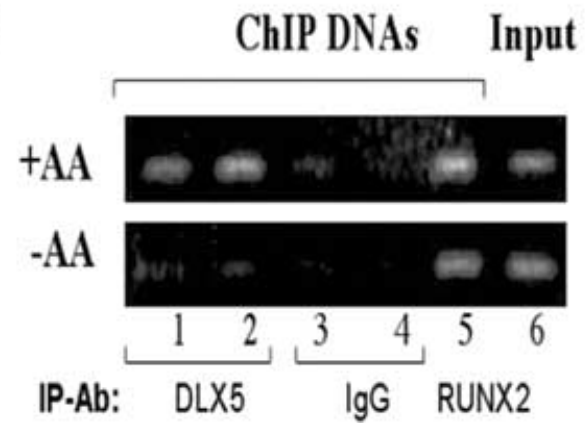

FIGURE 3. In vivo binding of RUNX2 and DLX5 to chromatin sites in differentiated and undifferentiated cells. (A) Schematic of the proximal $B s p$ promoter. RUNX2 and homeodomain (DLX5) protein-binding sites are indicated. (B) Comparison of Bsp chromatin occupancy by RUNX2 and DLX5. Chromatin immunoprecipitation (ChIP) assays were used to detect RUNX2 and DLX5 bound to the proximal Bsp promoter in control (-AA) and differentiated (+AA) MC3T3-E1 clone 4 cells. Antibodies used for ChIP are indicated. Note that RUNX2 remains chromatin associated regardless of differentiated state while $\mathrm{Dl} \times 5$ is only present in differentiated cells. From Roca et al., 2005. ${ }^{8}$

but RUNX2 mRNA and protein levels only increase 1.5 - to 2 -fold. Similarly, the amount of RUNX2 bound to the promoter of target genes as measured by chromatin immunoprecipitation also remains relatively unchanged during differentiation. ${ }^{7}$ FIGURE 3 shows RUNX2 bound to the proximal Bsp promoter. We identified two RUNX2 sites and a DLX5 homeodomain protein-binding site in this region and showed that these sites cooperate to control expression in osteoblasts. ${ }^{8}$ Surprisingly, when RUNX2 chromatin occupancy was compared in control versus differentiated osteoblasts, no obvious differences were observed. In contrast, DLX5 chromatin occupancy was only observed in differentiated cells. However, total DLX5 protein measured by Western blot was equivalent in control and differentiated cells indicating that the affinity of DLX5 for chromatin (and possibly Runx2) increases with differentiation.

There are at least two possible ways RUNX2 transcriptional activity could be regulated in the absence of changes in RUNX2 protein levels. First, as suggested by the DLX5 result discussed above, levels of cofactors or ability of cofactors to interact with RUNX2 could be regulated. Second, covalent modification could alter RUNX2 transcriptional activity. 
The first possibility is probably a very common control mechanism especially when we consider the broad range of factors known to interact with RUNX2. For example, CBF $\beta$ forms heterodimers with all members of the Runx family. ${ }^{9}$ RUNX2 also interacts with ATF4 and this factor may mediate some of the responses of osteoblasts to PTH. ${ }^{10,11}$ SMAD proteins, mediators of BMP/TGF $\beta$ actions, can also stimulate RUNX2 activity. ${ }^{12}$ In addition, there are a number of inhibitory factors. SOX9, mentioned above, suppresses RUNX2-dependent chondrocyte hypertrophy. ${ }^{4}$ TWIST may prevent RUNX2 stimulation of mineralization in developing cranial bones to prevent craniosynostosis. ${ }^{13}$ Last, histone deacetylases (HDACs) and HDAC accessory factors, such as $\mathrm{mSin} 3 \mathrm{a}$, are known to bind RUNX2 and keep chromatin in a deacetylated, inactive state. ${ }^{14}$

Posttranslational modification represents the second major mechanism for controlling RUNX2 activity. Over the past decade, we described a pathway involving integrin-mediated activation of the ERK/MAP kinase pathway that results in phosphorylation and stimulation of RUNX2 transcriptional activity. Integrins provide information to cells about the ECM environment and mediate cell attachment and spreading. ${ }^{15}$ Integrins are also major mediators of mechanical loads experienced by cells and may transduce information about ECM stiffness to control differentiation. ${ }^{16,17}$ In addition to providing continuity between the ECM and the actin-containing cytoskeleton, integrins are important signal transduction molecules that activate Ras-ERK and p38 MAP kinase pathways, calcium channels, and mechanosensors. ${ }^{15}$

Osteoblast differentiation requires elaboration of a collagenous ECM. This explains the well-known requirement for ascorbic acid in osteoblast differentiation since this vitamin cofactor is essential for secretion of the collagenous ECM. ${ }^{18}$ An example of ECM-mediated osteoblast differentiation was shown in FIGURE 2. Bone sialoprotein and osteocalcin, two osteoblast markers, are only expressed in cells grown in ascorbate-containing medium. In studies that will not be extensively discussed in this article, we showed that the response of osteoblasts to ECM is mediated by $\alpha 2 \beta 1$ integrins and the ERK/MAP kinase pathway. ${ }^{7} 19,20$ We specifically showed that osteoblast differentiation could be blocked with specific inhibitors of collagen synthesis and integrin-collagen binding. Furthermore, inhibition of MAPK signaling using either pharmacological inhibitors or dominant-negative pathway intermediates blocked differentiation. In contrast, induction of osteoblast gene expression was induced with a constitutively active MAPK intermediate, MEK1.

Initially, studies with the osteocalcin gene were used to relate MAPK responsiveness to RUNX2. We found that MAPK activation via transfection of cells with constitutively active MEK1 (MEK-SP) could induce the RUNX2responsive Ocn gene while dominant-negative MEK1 (MEK-DN) was inhibitory. ${ }^{20}$ An examination of the Ocn promoter for MAPK-responsive sequence elements identified two RUNX2-binding sites called OSE2a and b. 


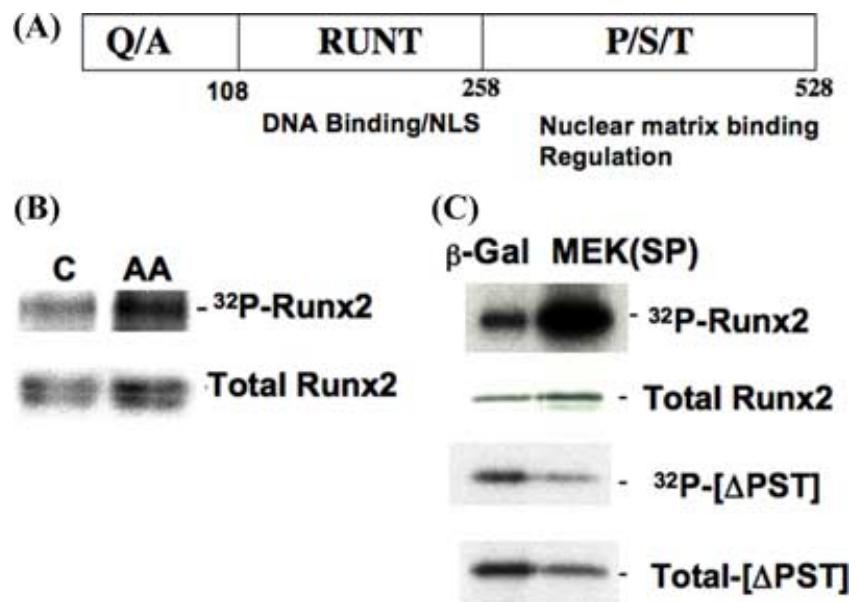

FIGURE 4. RUNX2 phosphorylation. (A) Cartoon showing major domains of RUNX2 and their known functions. (B) RUNX2 phosphorylation increases with osteoblast differentiation. MC3T3-E1 clone 4 cells were grown in control or differentiation (AA-containing) medium for 8 days before metabolic labeling with $\left[{ }^{32} \mathrm{P}\right]$ orthophosphate. RUNX2 was immunoprecipitated from nuclear extracts and visualized by autoradiography. Total RUNX2 was measured by Western blotting with RUNX2-specific antibody. (C) ERK/MAPK increases phosphorylation in the RUNX2 P/S/T domain. COS7 cells were transfected with RUNX2 and constitutively active MEK1 (MEK(SP)) expression vectors as indicated. RUNX2 phosphorylation was measured as in panel B. Phosphorylation of a truncated RUNX2 lacking the entire PST domain $(\triangle P S T)$ is also shown (bottom). Note lack of MEK(SP)-dependent phosphorylation. From Franceschi et al., 2003. ${ }^{28}$

Mutation of either of these sites reduced MAPK responsiveness with the proximal site, OSE2a, having the strongest effect on Ocn promoter activity. ${ }^{21}$

As might be expected, activation of MAPK, either by ascorbate addition or transfection of cells with MEK-SP results in increased RUNX2 phosphorylation (FIG. 4 and Ref. 20). Examination of different truncations of the RUNX2 protein showed that the $\mathrm{C}$-terminal proline/serine/threonine region $(\mathrm{P} / \mathrm{S} / \mathrm{T}$ domain) of RUNX2 was required for both MAPK responsiveness and phosphorylation (FIG. 4 and Ref. 20). More detailed deletional analysis further localized a minimal region for MAPK responsiveness between amino acids 254 and 320 (result not shown). The specific identification and functional significance of ERK/MAPK phosphorylation sites in RUNX2 will be reported separately.

A number of other stimuli that act through the ERK/MAPK pathway have been shown to stimulate RUNX2 phosphorylation and transcriptional activity. For example, we showed that FGF2 via its receptor, FGFR2, can rapidly stimulate ERK1/2 and RUNX2 phosphorylation and Ocn gene expression. ${ }^{22} \mathrm{~A}$ related study showed that $\mathrm{PKC} \delta$, a second kinase activated by the FGFR2, 
is also required for Ocn stimulation and that this kinase uses a distinct site in RUNX2 (S247) that can be resolved from ERK/MAPK sites. ${ }^{23}$ Last, exposure of osteoblasts to mechanical loading was shown to stimulate RUNX2 phosphorylation and osteoblast gene expression. ${ }^{24-26}$

All the studies discussed above were conducted in cell culture. To establish the importance of the ERK/MAPK pathway and RUNX2 phosphorylation to osteoblast function in vivo, we took a transgenic approach. ${ }^{27}$ Transgenes were constructed using a $0.6 \mathrm{~kb}$ mOG2 promoter to specifically drive MEK-SP and MEK-DN expression in osteoblasts. Transgene expression was detected only in bones and had a time course of expression during development that parallels expression of the endogenous Ocn gene. Examination of ERK1/2 phosphorylation in calvarial osteoblasts derived from these animals showed a $50 \%$ increase in cells from Mek-sp mice and a 50\% decrease in Mek-dn cells (see Ref. 27). This indicates that transgene expression leads to subtle changes in MAPK activity that resemble fluctuations normally induced by physiological stimuli. Examination of skeletal whole mounts of transgenic mice revealed that Mek- $d n$ decreased skeletal size and calvarial mineralization while these parameters were increased in Mek-sp mice (FIG. 5A-F). Histology of long bones revealed an additional interesting difference between wild-type and transgenic mice (FIG. 5G). At E15.5, long bones are normally undergoing endochondral ossification in diaphyseal regions. However, in Mek- $d n$ mice, this process is drastically delayed with only early bony collar formation being visible. In contrast, in the Mek-sp mice, endochondral bone formation is accelerated.

We next examined if transgenic modification of osteoblast MAPK activity could alter RUNX2 phosphorylation and transcriptional activity (FIG. 6). For this experiment, we isolated calvarial osteoblasts from transgenic mice and conducted metabolic labeling with $\left[{ }^{32} \mathrm{P}\right]$ orthophosphate followed by immunoprecipitation. Cells were also transfected with a Runx 2 reporter gene to measure transcriptional activity. Clearly, RUNX2 phosphorylation was increased in Mek-sp cells as was luciferase activity of the Ocn reporter gene. Also, as expected, Mek-sp stimulated in vitro osteoblast differentiation as measured by induction of osteoblast marker mRNAs or mineralization while differentiation was inhibited in Mek-sp cells (see Ref. 27).

To provide evidence that MAPK effects on skeletal development are mediated by RUNX2, we took a genetic approach. Runx2 +/- mice are known to have a characteristic phenotype (hypoplastic clavicles, patent fontanelles) that is a phenocopy of the human genetic disease, cleidocranial dysplasia or CCD. ${ }^{5}$ We reason that if MAPK acts by altering RUNX2 activity, calvaria and clavicles should be selectively sensitive to the Mek transgene when RUNX2 is limiting (i.e., in Runx $2+/-$ mice). To test this, Run $x 2+/-$ mice were crossed with Mek-sp or Mek-dn transgenic lines and the resulting skeletal phenotypes were examined at E19 (FIG. 7). Note the reduced calvarial mineralization and tiny clavicles in the Runx2 +/- embryos. The presence of the Mek-sp trans- 
(A)

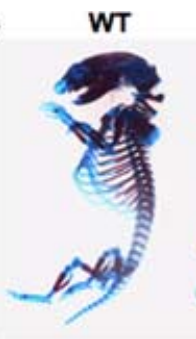

(B)

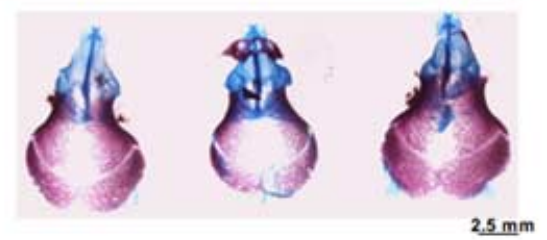

(C)
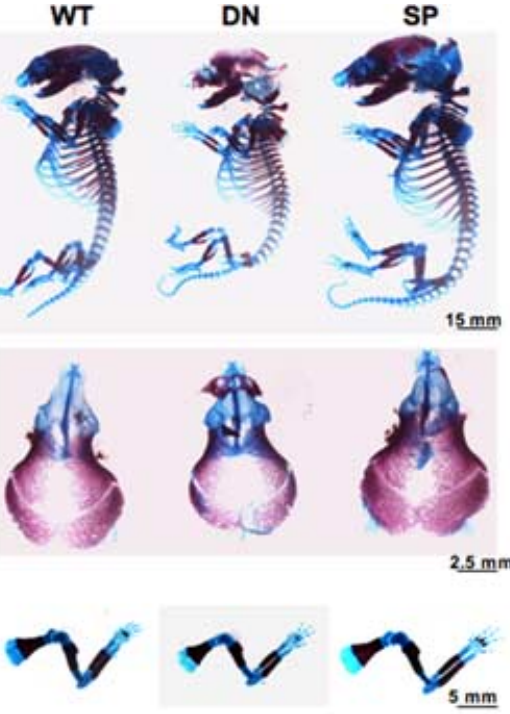

(D)

(E)
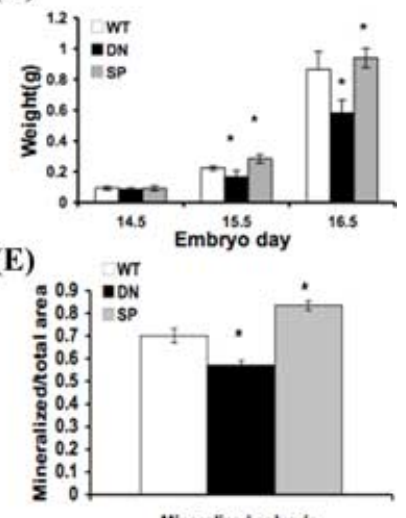

(F)

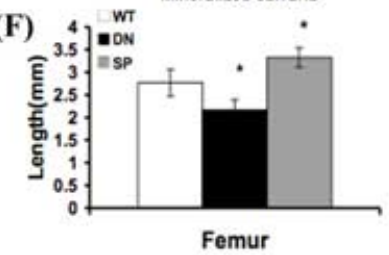

(G)

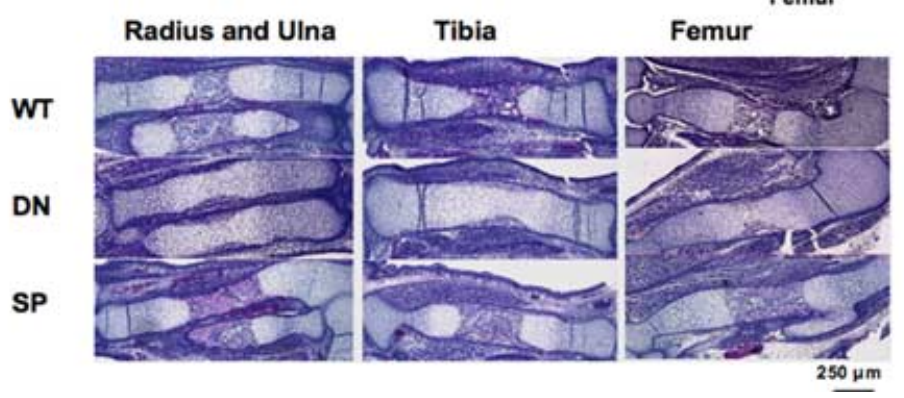

FIGURE 5. Altered skeletal development in TgMek- $d n$ and TgMek-sp mice. (A) Whole mounts of E15.5 skeletons stained with alcian blue and alizarin red. (D) Effects of transgene expression on embryo weights. (B, E) Cranial bones showing differences in mineralization (B) and quantification of mineralized area (expressed as percent of total calvarial area, E). (C, F) Hindlimbs showing differences in the size of bones with transgene expression (C) and quantification of femur lengths (F). (G) Histology of long bones from wild-type, $T g M e k-d n$ and TgMek-sp mice. Note delay in bony collar and trabecular bone in TgMek- $d n$ embryos. Bar $=250 \mu \mathrm{m}$. Statistical analysis values are expressed as means $\pm \mathrm{SD}, n=$ 8/group. * significantly different from wild-type at $P<0.01$. From Ge et al., 2007. ${ }^{27}$

gene led to a clear rescue of the CCD phenotype with increased clavicle size and calvarial mineralization. In the presence of Mek- $d n$, effects on Runx $2+/-$ mice were even more dramatic. In this case, the Mek-dn transgene exacerbated effects of Runx 2 haploinsufficiency with a further reduction in calvarial mineralization and near disappearance of clavicles. Notably, Runx2+/-; Mek- 
(A)

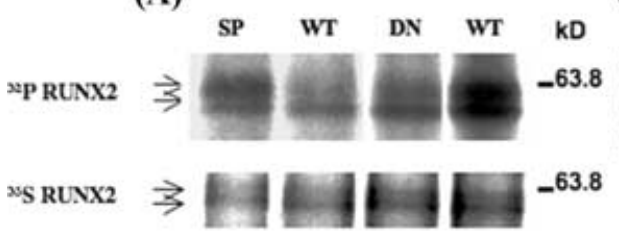

(B)
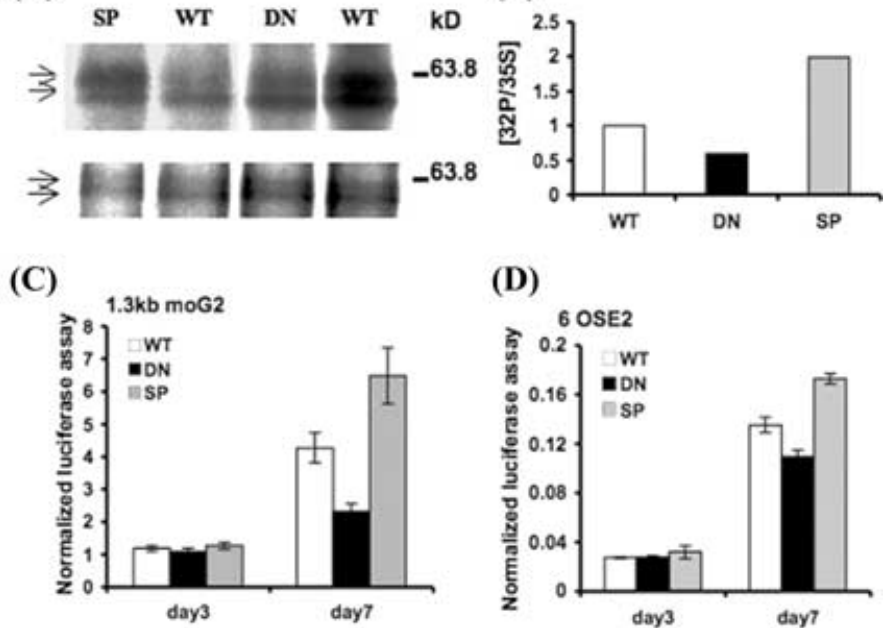

(D)

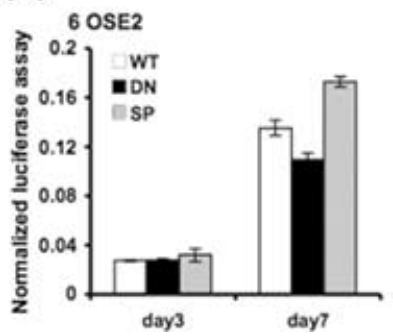

FIGURE 6. Changes in RUNX2 phosphorylation and transcriptional activity in osteoblasts from $T g M e k-d n$ and $T g M e k-s p$ mice. (A, B) Regulation of RUNX2 phosphorylation. Calvarial cells were grown under differentiating conditions for 10 days before metabolic labeling with $\left[{ }^{32} \mathrm{P}\right]$-orthophosphate or $\left[{ }^{35} \mathrm{~S}\right]$-methionine/cysteine (to normalize for total RUNX2) and immunoprecipitation with an anti-RUNX2 antibody. Each IP reaction contained $500 \mu \mathrm{g}$ total protein. Normalized ${ }^{32} \mathrm{P}$ incorporation into RUNX2 is shown in $\mathbf{B}$. (C, D) RUNX2-dependent transcriptional activity. Cells were transfected with p1.3mOG2luc $(\mathbf{C})$ or p6OSE2mOG2-luc (D) plasmids and grown under differentiating conditions for the times indicated before the measurement of luciferase activity. From Ge et al., 2007. ${ }^{27}$

$s p$ embryos did not survive the birth process due to severe skeletal defects. These experiments provide strong evidence that the ERK/MAPK pathway, via actions on RUNX2 transcriptional activity, is important for normal osteoblast differentiation and bone formation in vivo.

\section{SUMMARY}

Osteoblast differentiation is controlled by the sequential expression of several transcription factors. Among these is RUNX2, which is associated with commitment of mesenchymal cells to bone lineages and subsequent differentiation of osteoblasts and hypertrophic chondrocytes. RUNX2 is expressed throughout osteoblast and chondrogenic differentiation and appears to control different events at different stages of cell maturation. Rather than being controlled at transcriptional or translational levels, RUNX2 activity is controlled by its interaction with accessory factors and by posttranslational modifications, such as phosphorylation. Interaction of preosteoblasts with a type I collagencontaining ECM stimulates differentiation by an integrin-mediated pathway 
(A)

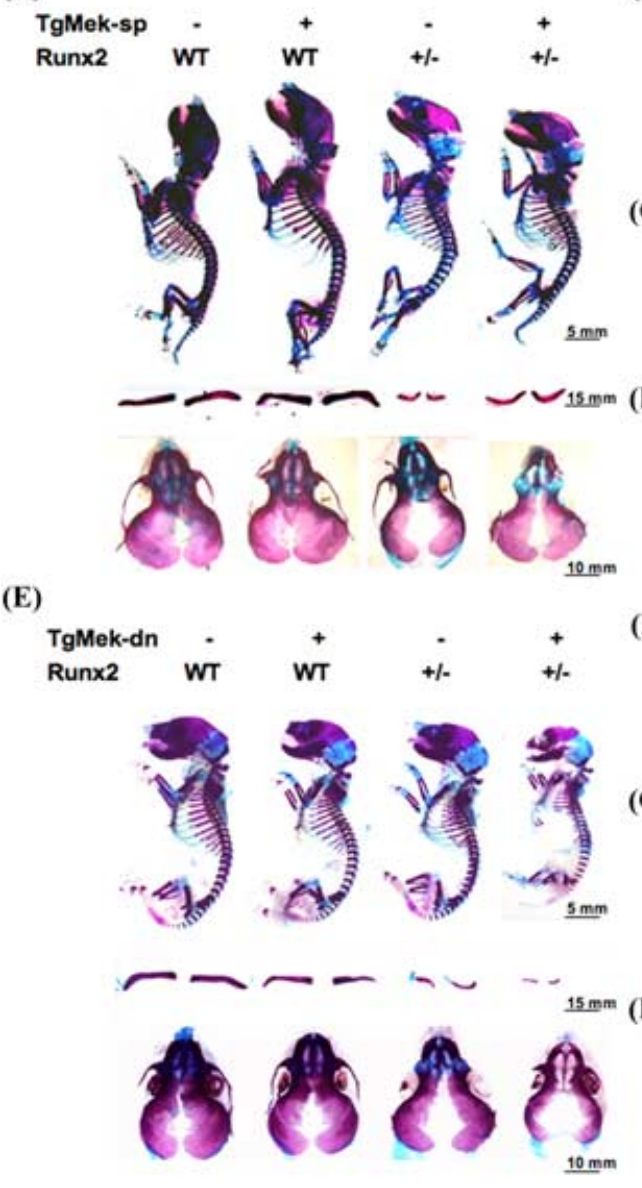

(B)

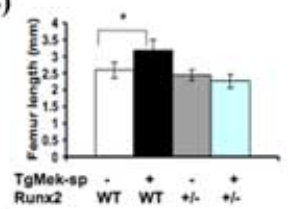

(C)

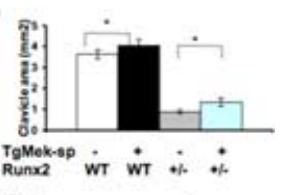

(D)

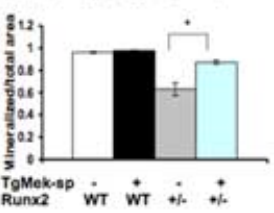

(F)

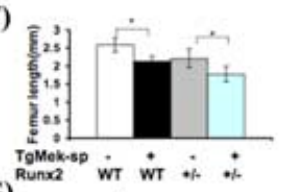

G)

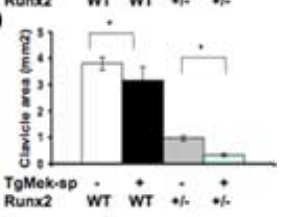

(H)

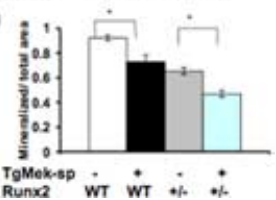

FIGURE 7. Genetic interactions between Mek- $d n$ and Mek-sp transgenes and Runx2. TgMek- $d n$ or TgMek-sp mice were crossed with Runx $2+/-$ mice to generate the genotypes indicated. (A-D) Partial rescue of CCD phenotype in Runx2+/- mice with Mek-sp. (A) Skeletal whole mounts of newborn mice stained with alcian blue and alizarin red (top), isolated clavicles (middle), and crania (bottom). (B-D) Measurements of femur length (B), clavicle areas, $(\mathbf{C})$ and mineralized area of calvaria (expressed as a fraction of total calvarial area). (E-H) Increased severity of CCD phenotype with Mek- $d n$. Groups are as in panels A-D. Statistical analysis values are expressed as means $\pm \mathrm{SD}, n=8$ /group. Comparisons are indicated by bars. ${ }^{*}$ significantly different at $P<0.01$. From Ge et al., $2007 .^{27}$

that, via activation of ERK/MAP kinase, phosphorylates RUNX2 in the $\mathrm{C}$-terminal $\mathrm{P} / \mathrm{S} / \mathrm{T}$ domain to increase transcriptional activity. This regulation, first identified in osteoblast cell cultures, also apparently occurs in vivo in that transgenic modification of ERK/MAPK activity affects skeletal development via a mechanism requiring RUNX2. 


\section{REFERENCES}

1. Ducy, P. et al. 1997. Osf2/Cbfa1: a transcriptional activator of osteoblast differentiation [see comments]. Cell 89: 747-754.

2. NAKASHIMA, K. et al. 2002. The novel zinc finger-containing transcription factor osterix is required for osteoblast differentiation and bone formation. Cell 108: 17-29.

3. YANG, X. et al. 2004. ATF4 is a substrate of RSK2 and an essential regulator of osteoblast biology; implication for Coffin-Lowry Syndrome. Cell 117: 387-398.

4. ZHOU, G. et al. 2006. Dominance of SOX9 function over RUNX2 during skeletogenesis. Proc. Natl. Acad. Sci. USA 103: 19004-19009.

5. Отто, F. et al. 1997. Cbfa1, a candidate gene for cleidocranial dysplasia syndrome, is essential for osteoblast differentiation and bone development. Cell 89: 765771.

6. Komori, T. et al. 1997. Targeted disruption of Cbfa1 results in a complete lack of bone formation owing to maturational arrest of osteoblasts. Cell 89: 755-764.

7. XIAO, G. et al. 1998. Role of the alpha2-integrin in osteoblast-specific gene expression and activation of the Osf2 transcription factor. J. Biol. Chem. 273: 32988-32994.

8. RocA, H. et al. 2005. Cooperative interactions between RUNX2 and homeodomain protein-binding sites are critical for the osteoblast-specific expression of the bone sialoprotein gene. J. Biol. Chem. 280: 30845-30855.

9. Iто, Y. 2004. Oncogenic potential of the RUNX gene family: 'overview'. Oncogene 23: 4198-4208.

10. XIAO, G. et al. 2005. Cooperative interactions between activating transcription factor 4 and Runx $2 / \mathrm{Cbfa} 1$ stimulate osteoblast-specific osteocalcin gene expression. J. Biol. Chem. 280: 30689-30696.

11. JIANG, D. et al. 2004. Parathyroid hormone induction of the osteocalcin gene. Requirement for an osteoblast-specific element 1 sequence in the promoter and involvement of multiple-signaling pathways. J. Biol. Chem. 279: 5329-5337.

12. AfZAL, F. et al. 2005. Smad function and intranuclear targeting share a Runx 2 motif required for osteogenic lineage induction and BMP2 responsive transcription. J. Cell. Physiol. 204: 63-72.

13. BialeK, P. et al. 2004. A twist code determines the onset of osteoblast differentiation. Dev. Cell. 6: 423-435.

14. IMAI, Y. et al. 2004. The corepressor mSin3A regulates phosphorylation-induced activation, intranuclear location, and stability of AML1. Mol. Cell Biol. 24: 1033-1043.

15. HyNES, R.O. 2002. Integrins: bidirectional, allosteric signaling machines. Cell 110: $673-687$.

16. You, J. et al. 2001. Osteopontin gene regulation by oscillatory fluid flow via intracellular calcium mobilization and activation of mitogen-activated protein kinase in MC3T3-E1 osteoblasts. J. Biol. Chem. 276: 13365-13371.

17. Engler, A.J. et al. 2006. Matrix elasticity directs stem cell lineage specification [see comment]. Cell 126: 677-689.

18. FRANCESCHI, R.T. 1992. The role of ascorbic acid in mesenchymal differentiation. Nutr. Rev. 50: 65-70.

19. XIAO, G. et al. 1997. Ascorbic acid-dependent activation of the osteocalcin promoter in MC3T3-E1 preosteoblasts: requirement for collagen matrix synthesis and the presence of an intact OSE2 sequence. Mol. Endocrinol. 11: 1103-1113. 
20. XIAO, G. et al. 2000. MAPK pathways activate and phosphorylate the osteoblastspecific transcription factor, Cbfa1. J. Biol. Chem. 275: 4453-4459.

21. FRENDO, J.L. et al. 1998. Functional hierarchy between two OSE2 elements in the control of osteocalcin gene expression in vivo. J. Biol. Chem. 273: 30509-30516.

22. XIAO, G. et al. 2002. Fibroblast growth factor 2 induction of the osteocalcin gene requires MAPK activity and phosphorylation of the osteoblast transcription factor, Cbfa1/Runx2. J. Biol. Chem. 277: 36181-36187.

23. KIM, H.J. et al. 2003. The protein kinase C pathway plays a central role in the fibroblast growth factor-stimulated expression and transactivation activity of Runx2. J. Biol. Chem. 278: 319-326.

24. WANG, F.S. et al. 2002. Superoxide mediates shock wave induction of ERKdependent osteogenic transcription factor (CBFA1) and mesenchymal cell differentiation toward osteoprogenitors. J. Biol. Chem. 277: 10931-10937.

25. ZIROS, P.G. et al. 2002. The bone-specific transcriptional regulator Cbfa1 is a target of mechanical signals in osteoblastic cells. J. Biol. Chem. 277: 23934-23941.

26. KANNO, T. et al. 2007. Mechanical stress-mediated Runx2 activation is dependent on Ras/ERK1/2 MAPK signaling in osteoblasts. J. Cell. Biochem. 101: 12661277.

27. GE, C. et al. 2007. Critical role of the extracellular signal-regulated kinase-MAPK pathway in osteoblast differentiation and skeletal development. J. Cell. Biol. 176: 709-718.

28. FrancesChI, R.T. et al. 2003. Multiple signaling pathways converge on the Cbfa1/Runx2 transcription factor to regulate osteoblast differentiation. Connect. Tissue Res. 44(Suppl 1): 109-116. 